\title{
Noninvasive measurement of dissipation in colloidal systems
}

\author{
B. Lander, ${ }^{1}$ J. Mehl, ${ }^{2}$ V. Blickle, ${ }^{2,3}$ C. Bechinger, ${ }^{2,3}$ and U. Seifert ${ }^{1}$ \\ ${ }^{1}$ II. Institut für Theoretische Physik, Universität Stuttgart, Pfaffenwaldring 57, 70550 Stuttgart, Germany \\ 2. Physikalisches Institut, Universität Stuttgart, Pfaffenwaldring 57, 70569 Stuttgart, Germany \\ ${ }^{3}$ Max-Planck-Institute for Intelligent Systems, Heisenbergstrasse 3, 70569 Stuttgart, Germany
}

(Received 30 July 2012; published 20 September 2012)

\begin{abstract}
According to Harada and Sasa [Phys. Rev. Lett. 95, 130602 (2005)], heat production generated in a nonequilibrium steady state can be inferred from measuring response and correlation functions. In many colloidal systems, however, it is a nontrivial task to determine response functions, whereas details about spatial steady state trajectories are easily accessible. Using a simple conditional averaging procedure, we show how this fact can be exploited to reliably evaluate average heat production. We test this method using Brownian dynamics simulations, and apply it to experimental data of an interacting driven colloidal system.
\end{abstract}

DOI: 10.1103/PhysRevE.86.030401

Introduction. The phenomenon of dissipation distinguishes equilibrium from nonequilibrium systems. For macroscopic systems, dissipation can be inferred either directly through measuring temperature changes or from the known external work applied to a system. At least for nonequilibrium steady states (NESSs) the latter is equal to the dissipation. For small systems such as colloidal particles or molecular motors, measuring dissipation is highly nontrivial. Calorimetric methods on the single particle or molecule level fail due to the tiny values of the heat generated by single degrees of freedom. In principle, the framework of stochastic thermodynamics [1-3] allows to apply the first law to phenomena on this scale from which the exchanged heat could be extracted if both the externally applied work to drive the system and the internal energy change could be measured, where the latter vanishes in a NESS. In practice, however, knowing the external force applied to a colloidal particle by a laser field beyond the paradigmatic harmonic trap [4] is quite a challenge $[5,6]$. Likewise, measuring directly the amount of adenosine triphosphate (ATP) molecules hydrolyzed by a single molecular motor is impossible. Thus, the ingredients of using the first law to infer dissipation, in general, are not directly accessible.

Harada and Sasa suggested an exact relation quantifying heat production in terms of the violation of the fluctuationdissipation relation (FDR) for systems with a single degree of freedom obeying overdamped Langevin dynamics [7]. Extensions to many-body systems both for over- and underdamped Langevin dynamics [8], as well as Hamiltonian systems [9], followed shortly after. Their results have been experimentally tested for an optically driven colloidal system [10] and applied to gain information about the nonequilibrium energetics of $F_{1}$-ATPase [11]. Further generalizations and experiments applying this technique comprise Langevin systems including memory [12,13], quantum Langevin dynamics [14], and FDR violations involving field variables [15]. For molecular motors connected to a colloidal probe, another approach has been put forward, from which information about dissipation can be deduced [16,17].

Specifically, the Harada-Sasa relation expresses the average heat production rate as

$$
\langle\dot{q}\rangle=\sum_{i=1}^{n} \mu_{i}^{-1}\left\{\left(v_{i}^{s}\right)^{2}+\int_{-\infty}^{\infty}\left[\tilde{C}_{i i}(\omega)-2 T \tilde{R}_{i i}^{\prime}(\omega)\right] \frac{d \omega}{2 \pi}\right\},
$$

PACS number(s): 82.70.Dd, 05.70.Ln

where Boltzmann's constant is set to unity, $T$ is the temperature of the surrounding heat bath, and $\mu_{i}$ is the mobility of the $i$ th of the $n$ degrees of freedom. The response function

$$
R_{i j}(t) \equiv \frac{\delta\left\langle\dot{x}_{i}(t)\right\rangle}{\delta f_{j}(0)}
$$

quantifies the change of the velocity $\dot{x}_{i}$ to a force perturbation applied to the $j$ th degree of freedom. Furthermore,

$$
C_{i j}(t) \equiv\left\langle\left[\dot{x}_{i}(t)-v_{i}^{\mathrm{s}}\right]\left[\dot{x}_{j}(0)-v_{j}^{\mathrm{s}}\right]\right\rangle
$$

is the velocity autocorrelation function, and $\tilde{g}(\omega) \equiv$ $\int_{-\infty}^{\infty} g(t) \exp (i \omega t) d t /(2 \pi)$ is the Fourier transform of an arbitrary function $g(t)$. The prime in Eq. (1) denotes the real part of a complex-valued function.

This exact relation yields insight into the origins of FDR violations and can be used readily if response functions are accessible. Determining response functions for driven colloidal systems, however, often poses a nontrivial and time-consuming task due to the need to perturb the system from its steady state. The perturbation must be sufficiently small to stay within the linear response regime and has to be applied to each degree of freedom separately. Determining response functions in frequency space requires separate measurements for each frequency, which must be done up to sufficiently high frequencies to make sure that the integral in Eq. (1) converges. If determined in temporal space, the system must be observed during its relaxation, which excludes the possibility to average over the time coordinate, thus substantially increasing the statistical effort.

In experiments and simulations, good statistics for trajectories in a NESS is readily available. In the following, we will employ a conditional averaging procedure on these trajectories from which the mean local velocity field can be obtained. Combined with the measured stationary distribution, this field then yields the average heat production rate. This procedure avoids difficulties arising from the application of external perturbations and reduces the effort to create the statistics needed. It thus complements the approach given by Harada and Sasa by making the average heat production rate easily accessible from steady state trajectories. Likewise, it does not require any knowledge about the applied forces or interactions within the system. 
Theory. We introduce this method for systems whose dynamics is governed by a set of coupled overdamped Langevin equations

$$
\dot{\mathbf{x}}=\mu \mathbf{F}(\mathbf{x})+\zeta,
$$

with the coordinate $\mathbf{x}=\left(x_{1}, \ldots, x_{n}\right)$, and the force $\mathbf{F}$ composed of conservative and nonconservative contributions. Interactions with the surrounding solvent are modeled by Gaussian white noise $\zeta$ with zero mean $\langle\zeta(t)\rangle=0$ and correlations

$$
\left\langle\zeta(t) \zeta^{T}\left(t^{\prime}\right)\right\rangle=2 \mu T \delta\left(t-t^{\prime}\right) .
$$

The stationary probability distribution function $p^{\mathrm{s}}(\mathbf{x})$ follows from the Smoluchowski equation according to

$$
0=-\nabla \cdot \mathbf{j}^{\mathrm{s}}(\mathbf{x}),
$$

with the probability current

$$
\mathbf{j}^{\mathrm{s}}(\mathbf{x}) \equiv \mu \mathbf{F}(\mathbf{x}) p^{\mathrm{s}}(\mathbf{x})-\mu T \nabla p^{\mathrm{s}}(\mathbf{x}) .
$$

The closely related mean local velocity is defined as the conditional average of the fluctuating velocity $\dot{\mathbf{x}}$ at position $\mathbf{x}$,

$$
\boldsymbol{v}^{\mathrm{s}}(\mathbf{x}) \equiv \lim _{\Delta t \rightarrow 0}\langle\mathbf{x}(t+\Delta t)-\mathbf{x}(t-\Delta t) \mid \mathbf{x}(t)=\mathbf{x}\rangle /(2 \Delta t) .
$$

Here, the Stratonovich convention has to be employed, thus evaluating the spatial variable in the midstep position. For the remainder of this Rapid Communication, we will always assume this convention. This relation enables us to evaluate the mean local velocity from NESS trajectories. Carrying out the average analytically, one obtains [1]

$$
\boldsymbol{v}^{\mathrm{s}}(\mathbf{x})=\mathbf{j}^{\mathrm{s}}(\mathbf{x}) / p^{\mathrm{s}}(\mathbf{x})=\mu \mathbf{F}(\mathbf{x})-\mu T \nabla \ln p^{\mathrm{s}}(\mathbf{x}) .
$$

The average heat production rate along a stochastic trajectory $\mathbf{x}(t)$ is given by [2]

$$
\langle\dot{q}\rangle \equiv\langle\mathbf{F}(\mathbf{x}(t)) \cdot \dot{\mathbf{x}}(t)\rangle .
$$

Since $\mathbf{x}$ is a stochastically fluctuating quantity, some care needs to be taken while averaging. In the Stratonovich scheme, one has [1]

$$
\langle\dot{q}\rangle=\left\langle\mathbf{F}(\mathbf{x}) \cdot v^{\mathrm{s}}(\mathbf{x})\right\rangle .
$$

Now, all fluctuating quantities have been replaced. Using Eq. (9) we substitute $\mathbf{F}$ and obtain

$\langle\dot{q}\rangle=\mu^{-1} \int d \mathbf{x} \boldsymbol{v}^{\mathrm{s}}(\mathbf{x})^{2} p^{\mathrm{s}}(\mathbf{x})+T \int d \mathbf{x} \boldsymbol{v}^{\mathrm{s}}(\mathbf{x}) \cdot \nabla p^{\mathrm{s}}(\mathbf{x})$.

The second integral on the right-hand side vanishes, since

$$
\begin{aligned}
\int d \mathbf{x} \boldsymbol{v}^{\mathrm{s}}(\mathbf{x}) \cdot \nabla p^{\mathrm{s}}(\mathbf{x}) & =\int d \mathbf{x} \mathbf{j}^{\mathrm{s}}(\mathbf{x}) \cdot \nabla \ln p^{\mathrm{s}}(\mathbf{x}) \\
& =-\int d \mathbf{x}\left[\nabla \cdot \mathbf{j}^{\mathrm{s}}(\mathbf{x})\right] \ln p^{\mathrm{s}}(\mathbf{x})=0 .
\end{aligned}
$$

Here, we have used Eq. (6) after a partial integration. The boundary term vanishes due to the periodicity of $\mathbf{j}^{\mathrm{s}}$ and $p^{\mathrm{s}}$. Therefore, the average heat production rate reads

$$
\langle\dot{q}\rangle=\mu^{-1} \int d \mathbf{x} \boldsymbol{v}^{\mathrm{s}}(\mathbf{x})^{2} p^{\mathrm{s}}(\mathbf{x})=\mu^{-1}\left\langle\boldsymbol{v}^{\mathrm{s}}(\mathbf{x})^{2}\right\rangle .
$$

Given a long trajectory $\mathbf{x}(t)$, we can determine the stationary distribution $p^{\mathrm{s}}$ and via Eq. (8) also the mean local velocity $\boldsymbol{v}^{\mathrm{s}}$. Therefore, we are able to evaluate the average heat production

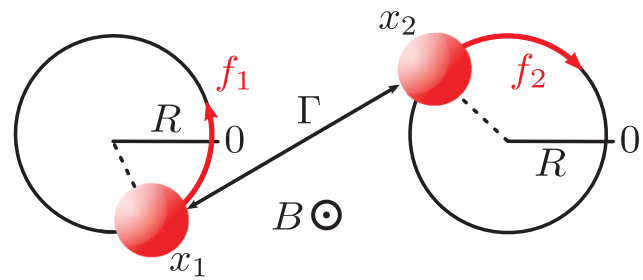

FIG. 1. (Color online) Schematic representation of the system: Two paramagnetic colloidal particles driven along two rings of radius $R$ by constant forces $f_{i}$. The position $x_{i}$ of the $i$ th particle is the arc length measured in the counterclockwise direction. The magnetic field applied perpendicular to the rings induces a magnetic moment in each particle. The strength of the resulting repulsive interaction is quantified by the dimensionless plasma parameter $\Gamma$.

rate in the system simply by recording particle trajectories in the NESS.

In the following, we will first illustrate the validity and usefulness of the method by using Brownian dynamics simulations to model a driven system consisting of two coupled colloidal particles. Second, we apply the method to data for the experimental system and determine the average heat production rate as a function of the coupling strength.

Simulation. We use Brownian dynamics simulations to test this method for the following, experimentally accessible [18], colloidal system. Two paramagnetic colloidal particles are driven on two nonoverlapping rings of radius $R$ (see Fig. 1). By applying a small homogeneous magnetic field $B$ normal to the plane containing the rings, the particles acquire parallel magnetic dipole moments $m \approx \alpha B$ with $\alpha \simeq 5.9 \times 10^{-12} \mathrm{~A} \mathrm{~m}^{2} / \mathrm{T}$. The resulting repulsive interaction is described by the potential $W(\mathbf{x})=\mu_{0} m^{2} /\left[\pi r^{3}(\mathbf{x})\right]$, where $\mu_{0}$ is the magnetic constant and $r(\mathbf{x})$ the distance between the particles. In order to quantify the interaction strength, we introduce the dimensionless plasma parameter $\Gamma \equiv \Delta W / T$, where $\Delta W$ is the difference between the maximum and minimum in the interaction energy. Apart from the interaction, the total force acting on the particle on the $i$ th ring,

$$
F_{i}(\mathbf{x})=f_{i}-\partial_{x_{i}}\left[V_{i}\left(x_{i}\right)+W(\mathbf{x})\right],
$$

contains the sinusoidal potential $V_{i}=A_{i} \sin \left(x_{i} / R-\phi_{i}\right)$ of amplitude $A_{i}$ and phase shift $\phi_{i}$, and the constant driving force $f_{i}$. Using these forces, the dynamics of the system is given by the Langevin equation (4). The equations of motion are integrated via a stochastic Runge-Kutta algorithm [19] using a time step of $\Delta t=0.0001(\mu \mathrm{m})^{2} /(\mu \mathrm{T})$.

The most crucial point of our method is the determination of the mean local velocity $\boldsymbol{v}^{\mathrm{s}}$ and the probability distribution function $p^{\mathrm{s}}$. In order to check this point, we proceed as follows. First, we compute the force field $\mathbf{F}$ analytically, which is possible since in this simulation all potential parameters are known in detail. Second, we compute $\mathbf{F}$ via Eq. (9) by evaluating both $\boldsymbol{v}^{\mathrm{s}}$ and the gradient field of $p^{\mathrm{s}}$ from the simulated data. In Fig. 2, we compare the differently obtained force fields and find very good agreement.

We calculate the average heat production rate using two different methods: (A) We use the trajectory-based method 

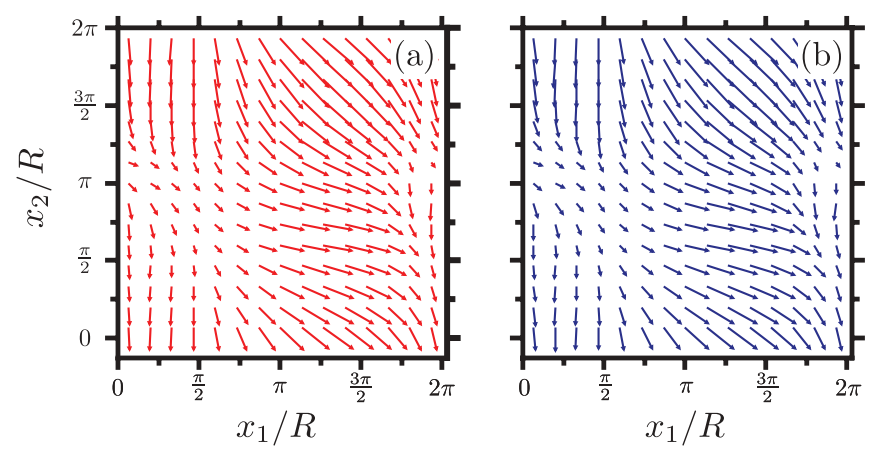

FIG. 2. (Color online) Comparison between the force fields $\mathbf{F}(\mathbf{x})$ for the two-ring system obtained (a) analytically and (b) from NESS trajectories via Eq. (9) for parameter set $I$ (see Table I).

introduced in this Rapid Communication, i.e., we determine $\boldsymbol{v}^{\mathrm{s}}$ and $p^{\mathrm{s}}$ from the simulated trajectories and use Eq. (14) to obtain $\langle\dot{q}\rangle$. (B) As a test, we determine $\langle\mathbf{F} \cdot \dot{\mathbf{x}}\rangle$ along the complete trajectory for each particle. The sum then yields the true average heat production rate in this simulation run. Of course, this procedure is possible only if details of the interaction are known, as is the case in these Brownian dynamics simulations.

In Fig. 3, we present a comparison between the differently evaluated average heat production rates determined for three different parameter sets (see Table I). We find very good agreement, supporting the consistency between the present approach (A) and the reference result (B). The most critical point in the application of method (A) is the determination of $\boldsymbol{v}^{\mathrm{s}}$ in those phase-space regions where the system is found with low probability. The average heat production rate $\langle\dot{q}\rangle$, however, is computed from an integral [see Eq. (14)] in which $\left(v^{\mathrm{s}}\right)^{2}$ enters weighted by $p^{\mathrm{s}}$. Since in rarely visited regions
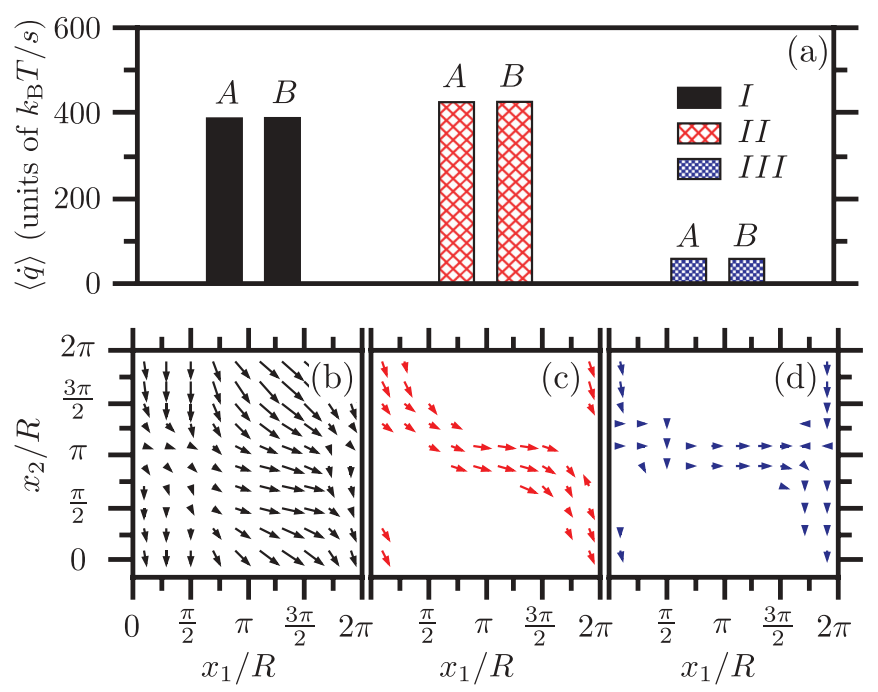

FIG. 3. (Color online) (a) Average heat production rates $\langle\dot{q}\rangle$ determined from methods (A) and (B) as described in the text for three different parameter sets I, II, and III (see Table I). To relate $\langle\dot{q}\rangle$ to an experimental scale we choose $a=5.2 \mu \mathrm{m}$. Error bars are smaller than $1 \%$.(b)-(d) Corresponding evaluated mean local velocity fields $\boldsymbol{v}^{\mathrm{s}}(\mathbf{x})$. Regions without arrows are rarely visited by the system.
TABLE I. Parameter sets I, II, III.

\begin{tabular}{lrrr}
\hline \hline & \multicolumn{1}{c}{ I } & \multicolumn{1}{c}{ II } & \multicolumn{1}{c}{ III } \\
\hline$\Gamma$ & 310.0 & 670.0 & 310.0 \\
$f_{1}\left(k_{\mathrm{B}} T / \mu \mathrm{m}\right)$ & 50.0 & 56.0 & 25.0 \\
$f_{2}\left(k_{\mathrm{B}} T / \mu \mathrm{m}\right)$ & -65.0 & -54.0 & -35.0 \\
$A_{1}\left(k_{\mathrm{B}} T\right)$ & 225.0 & 175.0 & 175.0 \\
$A_{2}\left(k_{\mathrm{B}} T\right)$ & 175.0 & 170.0 & 175.0 \\
$\phi_{1}$ & $-\pi / 5$ & $-\pi / 5$ & $-\pi / 5$ \\
$\phi_{2}$ & $-3 \pi / 5$ & $-3 \pi / 5$ & $-3 \pi / 5$ \\
$R(\mu \mathrm{m})$ & 3.5 & 3.5 & 3.5 \\
Center-to-center distance $(\mu \mathrm{m})$ & 17.0 & 17.0 & 17.0 \\
\hline \hline
\end{tabular}

this weight is small, their contributions to $\langle\dot{q}\rangle$ should play a minor role, provided that $\boldsymbol{v}^{\mathrm{s}}$ stays finite. This is the case in our system, and should hold for many other well-behaved systems as well. Therefore, to obtain averaged quantities such as $\langle\dot{q}\rangle$, only regions in configuration space of substantial weight must be sampled accurately. Regions of minor probability will only add small-sized corrections to the result.

Experiment. Having demonstrated its validity, we now apply the method to the experimental system [18]. Two paramagnetic colloidal particles with diameters $a=5.2 \mu \mathrm{m}$ are driven by a scanning laser beam [20,21] along two rings with radii $R=3.5 \mu \mathrm{m}$ and a center-to-center distance of $17 \mu \mathrm{m}$. We checked for hydrodynamic interactions by measuring the mean velocity of a particle driven along a circle with constant potential in the presence of another particle at minimal distance $d$. For $d \geqslant 9 \mu \mathrm{m}$, we do not find any deviation from the $d \rightarrow \infty$ limit. We thus conclude that hydrodynamic interactions are negligible in this case. ${ }^{1}$ Using digital video microscopy, we track the NESS trajectories $x_{1}(t)$ and $x_{2}(t)$ with a spatial and temporal resolution of $20 \mathrm{~nm}$ and $25 \mathrm{~ms}$, respectively. We determine the average heat production rate for different magnetic fields, $B \leqslant 40 \mathrm{mT}$, thus changing the plasma parameter in the range of $0 \leqslant \Gamma \leqslant 1100$.

In the absence of coupling, the particles move independently along their tilted potentials with a mean circulation time of $12 \mathrm{~s}$. This motion leads on average to a heat production rate of $\langle\dot{q}\rangle=\mu^{-1}\left\langle\boldsymbol{v}^{\mathrm{s}}(\mathbf{x})^{2}\right\rangle=199 k_{\mathrm{B}} T / \mathrm{s}$ with $\mu^{-1}=3 \pi \eta a$ the bare mobility of one particle and $\eta$ the viscosity of the solvent. Under strong coupling conditions, $\langle\dot{q}\rangle$ reaches higher values [see Fig. 4(a)]. This fact becomes obvious when focusing on the corresponding mean local velocity fields $\boldsymbol{v}^{\mathrm{s}}$. For $\Gamma=0$, $\boldsymbol{v}^{\mathrm{s}}$ shows a large region with very small velocities [see Fig. 4(b)] corresponding to potential minima at $x_{1} / R=\pi / 4$ and $x_{2} / R=3 \pi / 4$. For $\Gamma \geqslant 480$, the motion of the two particles synchronizes due to the repulsive interaction as follows [see Fig. 4(c)]. While the first particle fluctuates around its

\footnotetext{
${ }^{1}$ Additionally, we have estimated the strength of the hydrodynamic coupling in relation to the dipole interaction up to the Rotne-Prager level. Comparing the contributions of the different types of interaction in the range of angles where the particles come closest, we find that hydrodynamic contributions are $\lesssim 20 \%$ of the dipolar contribution for the smallest nonzero $\Gamma$ used and smaller by at least one order of magnitude for all others.
} 

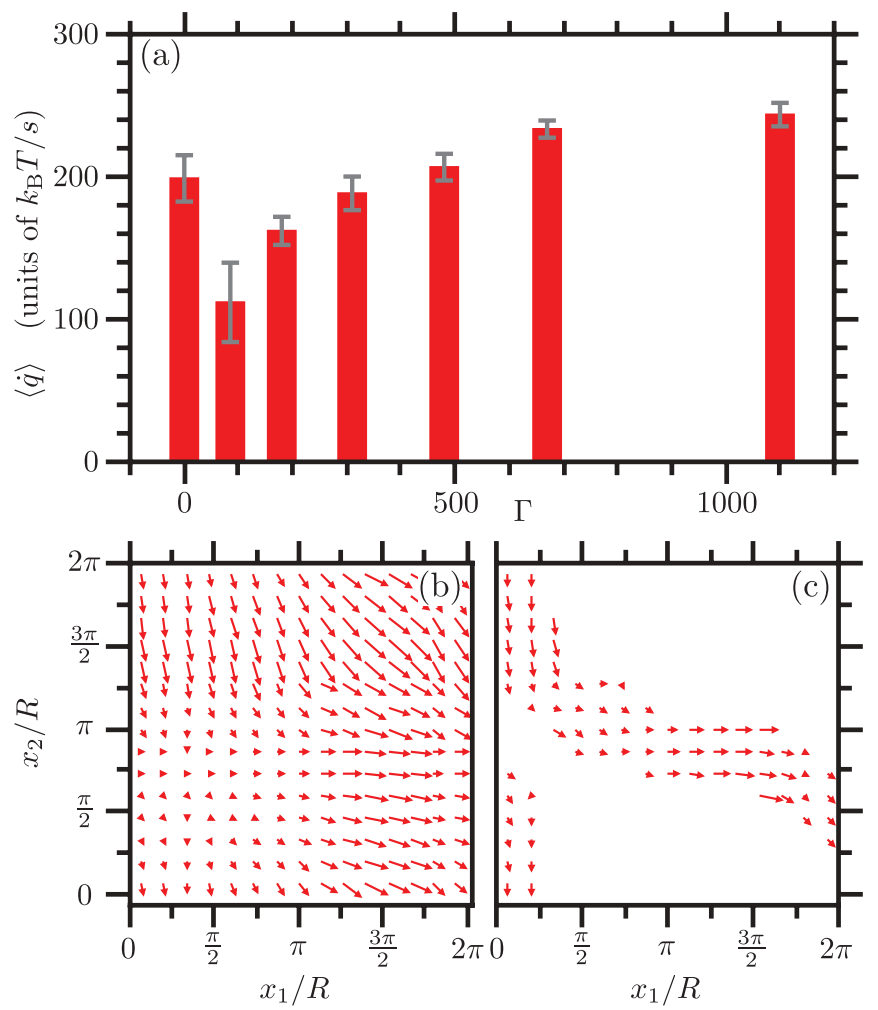

FIG. 4. (Color online) (a) Average heat production rates $\langle\dot{q}\rangle$ for different plasma parameters $\Gamma$. The parameters for the NESSs are $f_{1}=56 k_{\mathrm{B}} T / \mu \mathrm{m}, A_{1}=175 k_{\mathrm{B}} T$, and $f_{2}=-51 k_{\mathrm{B}} T / \mu \mathrm{m}, A_{1}=$ $164 k_{\mathrm{B}} T$ obtained by the method presented in Ref. [22]. Error bars are obtained by evaluating the data set for four parts of equal size and computing the standard deviation. (b), (c) Corresponding mean local velocity fields $\boldsymbol{v}^{\mathrm{s}}(\mathbf{x})$ determined from the NESS trajectories for plasma parameters (b) $\Gamma=0$ and (c) $\Gamma=1150$, respectively. potential minimum, the second one completes one circulation. Arriving at the point where the particles are closest, this particle pushes the first one over its barrier. Then, the roles switch and the first particle completes a circulation while the second one fluctuates around its potential minimum. This effect shortens the time to overcome the potential barrier considerably for both particles and results, on average, in a faster circulation, and thus a larger $\langle\dot{q}\rangle$ than in the uncoupled case. In the intermediate regime, the particles may still overtake one another. During the overtaking process, the faster particle hinders the other one in overcoming the potential barrier, effectively slowing down its motion. This on average slower motion produces less heat, and consequently $\langle\dot{q}\rangle$ is nonmonotonic in $\Gamma$ [see Fig. 4(a)]. We emphasize that we obtained robust results for $\langle\dot{q}\rangle$ for NESS trajectories with a length of only $30 \mathrm{~min}$ corresponding to 150 full revolutions of each particle.

Concluding perspectives. In this Rapid Communication, we have presented an approach to determine the average heat production rate for colloidal systems in a NESS. While the Harada-Sasa method quantifies dissipation via response and correlation functions, the present method solely uses information obtained from steady state trajectories without the need to perturb the system. Neither does the method require any information about the underlying potentials or driving forces. Recording particle trajectories in the NESS is sufficient. This approach thus constitutes a complementary way to measure dissipation, which is easily implemented particularly in setups concerned with small colloidal systems. By replacing the stationary distribution function and the stationary mean local velocity field by their time-dependent counterparts [1], a generalization of the present method should also be applicable to relaxing and time-dependent systems.
[1] U. Seifert, Eur. Phys. J. B 64, 423 (2008); arXiv:1205.4176 [Rep. Prog. Phys. (to be published)].

[2] K. Sekimoto, Stochastic Energetics (Springer, Berlin, 2010).

[3] C. Jarzynski, Annu. Rev. Condens. Matter Phys. 2, 329 (2011).

[4] G. M. Wang, E. M. Sevick, E. Mittag, D. J. Searles, and D. J. Evans, Phys. Rev. Lett. 89, 050601 (2002).

[5] V. Blickle, T. Speck, L. Helden, U. Seifert, and C. Bechinger, Phys. Rev. Lett. 96, 070603 (2006).

[6] P. Jop, A. Petrosyan, and S. Ciliberto, Europhys. Lett. 81, 50005 (2008).

[7] T. Harada and S. I. Sasa, Phys. Rev. Lett. 95, 130602 (2005).

[8] T. Harada and S. I. Sasa, Phys. Rev. E 73, 026131 (2006).

[9] H. Teramoto and S. I. Sasa, Phys. Rev. E 72, 060102 (2005).

[10] S. Toyabe, H.-R. Jiang, T. Nakamura, Y. Murayama, and M. Sano, Phys. Rev. E 75, 011122 (2007).

[11] S. Toyabe, T. Okamoto, T. Watanabe-Nakayama, H. Taketani, S. Kudo, and E. Muneyuki, Phys. Rev. Lett. 104, 198103 (2010).
[12] J. M. Deutsch and O. Narayan, Phys. Rev. E 74, 026112 (2006).

[13] S. Toyabe and M. Sano, Phys. Rev. E 77, 041403 (2008).

[14] K. Saito, Europhys. Lett. 83, 50006 (2008).

[15] T. Harada, Phys. Rev. E 79, 030106 (2009).

[16] S. Toyabe, H. Ueno, and E. Muneyuki, Europhys. Lett. 97, 40004 (2012).

[17] E. Zimmermann and U. Seifert, arXiv:1209.3667 [New. J. Phys. (to be published)].

[18] J. Mehl, B. Lander, C. Bechinger, V. Blickle, and U. Seifert, Phys. Rev. Lett. 108, 220601 (2012).

[19] P. E. Kloeden, E. Platen, and H. Schurz, Numerical Solution of SDE Through Computer Experiments (Springer, Berlin, 2003).

[20] L. P. Faucheux, G. Stolovitzky, and A. Libchaber, Phys. Rev. E 51, 5239 (1995).

[21] J. Mehl, V. Blickle, U. Seifert, and C. Bechinger, Phys. Rev. E 82, 032401 (2010).

[22] V. Blickle, T. Speck, U. Seifert, and C. Bechinger, Phys. Rev. E 75, 060101 (2007). 